Acknowledgments. We thank Brian Fung, PharmD, Evan Draper, PharmD, Aaron Tande, MD, and the Mayo Clinic Enterprise Antimicrobial Stewardship Team. We dedicate this article to the memory of Dr James Steckelberg, a pioneer of antimicrobial stewardship.

Financial support. No financial support was provided relevant to this article.

Conflicts of interest. All authors report no conflicts of interest relevant to this article.

\section{References}

1. Stevens MP, Patel PK, Nori P. Involving antimicrobial stewardship programs in COVID-19 response efforts: all hands on deck. Infect Cont Hosp Epidemiol 2020 [Epub ahead of print]. doi: 10.1017/ice.2020.69.

2. Fung B, Draper E, Steckelberg J, et al. Conversion of a complex legacy antimicrobial stewardship clinical decision support system into epic. Poster presented at the AMIA 2019 Annual Symposium; November 18, 2019; Washington, DC.
3. Draper E, Fung B, Steckelberg J, et al. Customization of an antimicrobial stewardship clinical decision support module: reducing the noise and improving reporting. Poster presented at the AMIA 2019 Annual Symposium; November 19, 2019; Washington, DC.

4. Wilson JW, Oyen LJ, Ou NN, et al. Hospital rules-based system: the next generation of medical informatics for patient safety. Am J Health-Syst Pharm 2005;62:499-505.

5. Gautret P, Lagier JC, Parola P, et al. Hydroxychloroquine and azithromycin as a treatment of COVID-19: results of an open-label non-randomized clinical trial. Int J Antimicrob Agents 2020: [Epub ahead of print]. doi: 10.1016/ j.ijantimicag.2020.105949.

6. Gautret P, Lagier J-C, Parola P, et al. Clinical and microbiological effect of a combination of hydroxychloroquine and azithromycin in 80 COVID-19 patients with at least a six-day follow up: an observational study 2020 . Travel Med Infect Dis 2020 [Epub ahead of print]. doi: 10.1016/j.tmaid. 2020.101663 .

\title{
Positive RT-PCR tests among discharged COVID-19 patients in Shenzhen, China
}

\author{
Xiujuan Tang MMed ${ }^{1, a}$, Shi Zhao MPhil2,a ${ }^{\text {(1) }}$, Daihai He $\mathrm{PhD}^{3}$ (1), Lin Yang PhD , Maggie H. Wang PhD², Yuan Li MMed', \\ Shujiang Mei BS ${ }^{1}$ and Xuan Zou MMed ${ }^{1}$ \\ ${ }^{1}$ Shenzhen Center for Disease Control and Prevention, Shenzhen, China, ${ }^{2} \mathrm{JC}$ School of Public Health and Primary Care, Chinese University of Hong Kong, \\ Hong Kong, China, ${ }^{3}$ Department of Applied Mathematics, Hong Kong Polytechnic University, Hong Kong, China and ${ }^{4}$ School of Nursing, Hong Kong Polytechnic \\ University, Hong Kong, China
}

To the Editor-According to the current guideline of the National Health Commission of China, discharge of inpatients with the coronavirus 2019 (COVID-19) infection in China have to fulfill 2 recovery criteria: (1) symptoms disappear and computed tomography (CT) images become normal and (2) test negative for 2 consecutive times in reverse transcriptase-polymerase chain reaction (RT-PCR) tests for SARS-CoV-2. ${ }^{1}$ However, Lan et al $^{1}$ recently reported 4 cases who were tested positive for SARS-CoV-2 at 5 days after discharge, suggesting positive status among discharged patients. $^{2}$ To date, the prevalence and associated risk factors remain unclear.

We investigated all 209 patients with laboratory-confirmed SARS-CoV-2 infection who were discharged from the designated hospital in Shenzhen, China, between January 23 and February 21, 2020. Demographic data, laboratory profile, clinical data, and CT images were collected from these patients' electronic medical records. Throat swabs and anal swabs were collected from all patients for RT-PCR tests according to the following scenarios: (1) on February 18, 2020, for those discharged before February 12, 2019; (2) on February 19, 2020 for those discharged between

Author for correspondence: Shi Zhao, E-mail: zhaoshi.cmsa@gmail.com. Or Daihai He, E-mail: daihai.he@polyu.edu.hk. Or Shujiang Mei, E-mail: sjmei66@163.com. Or Xuan Zou, E-mail: 914494557@qq.com.

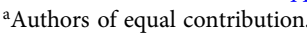

Cite this article: Tang X, et al. (2020). Positive RT-PCR tests among discharged COVID-19 patients in Shenzhen, China. Infection Control \& Hospital Epidemiology, 41: 1110-1112, https://doi.org/10.1017/ice.2020.134
February 13 and 19, 2019; (3) on days 7 and 14 after discharge thereafter. This study was approved by the Shenzhen Center for Disease Control and Prevention review board and the need for informed consent was waived. All data used in this work are available upon request and approval of Shenzhen Center for Disease Control and Prevention.

We compared the settings in the study by Lan et $\mathrm{al}^{2}$ with those in this study (Appendix Table S1 online). Logistic regression models were adopted to explore the factors associated with the RT-PCR test results. Odds ratios (ORs) were calculated for the probability of positive test in throat swabs, or anal swabs, or either, and the rest were considered negative in each of the 3 scenarios. The results are as follows:

- Scenario 1: 9 positive RT-PCR test results from throat swabs

- Scenario 2: 13 positive RT-PCR test results from anal swabs

- Scenario 3: 22 positive RT-PCR for test results from either throat or anal swabs

Normally, only scenario 3 should be considered, but we included scenario 1 to be consistent with Lan et al. ${ }^{2}$

Among all 209 discharged patients, 9 (4.3\%) tested positive in throat swabs only, 13 patients $(6.2 \%)$ tested positive in anal swabs only, and $22(10.5 \%)$ tested positive in either. Together, $10.5 \%$ of discharged patients showed virus shredding around an average of 4.7 days after discharge (range, 2-13 days). Under scenario 3, the logistic regression models revealed that a high risk of positive test

(C) 2020 by The Society for Healthcare Epidemiology of America. All rights reserved. This is an Open Access article, distributed under the terms of the Creative Commons Attribution licence (http://creativecommons.org/licenses/by/4.0/), which permits unrestricted re-use, distribution, and reproduction in any medium, provided the original work is properly cited. 
Table 1. Summary of the Characteristics of Study Patients and the Estimated Association Between the Individual Features and RT-PCR Testing Outcomes

\begin{tabular}{|c|c|c|c|c|c|}
\hline \multirow[b]{2}{*}{ Scenario 1} & & \multicolumn{4}{|c|}{ Throat Swabs } \\
\hline & & $\begin{array}{l}\text { Positive } \\
(N=9)\end{array}$ & $\begin{array}{l}\text { Negative } \\
(N=200)\end{array}$ & Crude OR & Adjusted $\mathrm{OR}^{\mathrm{a}}$ \\
\hline Sex & Male & 3 & 88 & $0.64(0.15-2.71)$ & $0.99(0.27-3.62)$ \\
\hline Age, y & Median (IQR) & $32(28-36)$ & $45(32-57)$ & $0.97(0.93-1.00)$ & $0.95(0.92-0.99)$ \\
\hline Sampling delay, d & Median (IQR) & $2(2-2)$ & $6(3-7)$ & $0.41(0.21-0.81)$ & $0.36(0.18-0.72)$ \\
\hline \multirow[t]{4}{*}{ Symptoms } & Dry cough & 4 & 72 & $1.42(0.36-5.65)$ & $1.38(0.44-4.32)$ \\
\hline & Expectoration & 2 & 27 & $1.83(0.35-9.67)$ & $3.03(0.61-15.14)$ \\
\hline & Cough & 5 & 89 & $1.56(0.39-6.19)$ & $1.56(0.52-4.70)$ \\
\hline & Diarrhea & 1 & 9 & $2.65(0.28-24.89)$ & $7.01(0.52-95.40)$ \\
\hline & & \multicolumn{4}{|c|}{ Anal Swabs } \\
\hline Scenario 2 & & $\begin{array}{l}\text { Positive } \\
(N=13)\end{array}$ & $\begin{array}{l}\text { Negative } \\
(N=196)\end{array}$ & Crude OR & Adjusted $\mathrm{OR}^{\mathrm{a}}$ \\
\hline Sex & Male & 5 & 86 & $0.80(0.25-2.61)$ & $0.83(0.28-2.46)$ \\
\hline Age, $y$ & Median (IQR) & $25(6-39)$ & $45(32-58)$ & $0.95(0.91-0.98)$ & $0.95(0.92-0.98)$ \\
\hline Sampling delay, d & Median (IQR) & $7(5-7)$ & $5(2-7)$ & $1.04(0.91-1.19)$ & $1.11(0.97-1.26)$ \\
\hline \multirow[t]{4}{*}{ Symptoms } & Dry cough & 6 & 70 & $1.54(0.48-4.91)$ & $1.92(0.65-5.63)$ \\
\hline & Expectoration & 3 & 26 & $1.96(0.49-7.87)$ & $3.00(0.67-13.37)$ \\
\hline & Cough & 8 & 86 & $2.05(0.63-6.67)$ & $3.12(0.97-10.07)$ \\
\hline & Diarrhea & 2 & 8 & $4.27(0.78-23.55)$ & $10.44(1.60-68.16)$ \\
\hline & & \multicolumn{4}{|c|}{ Either Throat or Anal Swabs } \\
\hline Scenario 3 & & $\begin{array}{c}\text { Positive } \\
(\mathrm{N}=22)\end{array}$ & $\begin{array}{l}\text { Negative } \\
(\mathrm{N}=187)\end{array}$ & Crude OR & Adjusted $\mathrm{OR}^{\mathrm{a}}$ \\
\hline Sex & Male & 8 & 83 & $0.72(0.28-1.83)$ & $0.70(0.29-1.66)$ \\
\hline Age, $y$ & Median (IQR) & $28(20-38)$ & $46(32-59)$ & $0.95(0.93-0.98)$ & $0.95(0.93-0.98)$ \\
\hline Sampling delay, d & Median (IQR) & $4(2-7)$ & $5(3-7)$ & $0.90(0.77-1.05)$ & $0.95(0.81-1.10)$ \\
\hline \multirow[t]{4}{*}{ Symptoms } & Dry cough & 10 & 66 & $1.53(0.61-3.81)$ & $1.89(0.81-4.39)$ \\
\hline & Expectoration & 5 & 24 & $2.00(0.66-6.08)$ & $4.00(1.24-12.88)$ \\
\hline & Cough & 13 & 81 & $1.89(0.75-4.75)$ & $2.70(1.12-6.51)$ \\
\hline & Diarrhea & 3 & 7 & $4.06(0.93-17.64)$ & $9.59(2.02-45.62)$ \\
\hline
\end{tabular}

Note. RT-PCR, reverse transcriptase-polymerase chain reaction; OR, odds ratio; IQR, interquartile range.

aThe $\mathrm{OR}$ is adjusted by the age, sex, sampling delay, disease severity and the backgrounds of the healthcare staff who delivered the treatment.

was significantly associated with older age (OR, 0.95 ; $95 \%$ confidence interval $[\mathrm{CI}], 0.93-0.98$ ), diarrhea during hospital stage (OR, 10.44; 95\% CI, 1.60-68.16). The "during disease" stage was the other significant factor, with an adjusted and $9.59(95 \%$ CI, 2.02-45.62) under scenarios 2 and 3, respectively. Expectoration during the disease stage is also a significant factor, with an adjusted OR of 4.00 (95\% CI, 1.24-12.88) but only under scenario 3 (Table 1).

Although the prevalence of virus was substantial (10.5\%), no infection was discovered among close contacts. Discharged COVID-19 patients in Shenzhen are required to be self-isolated for an additional 14 days after discharge to prevent the possible transmission due to the positive test post discharge.

Although live SARS-CoV-2 virus has been found in stool samples in some cases, ${ }^{3}$ the role of fecal-oral transmission remains unclear. Among 209 patients, 10 (4.8\%) had diarrhea, and this ratio is slightly higher than the $3.8 \%$ rate based on 1,099 patients nationwide, ${ }^{4}$ and 2 of 10 patients (20\%) with diarrhea showed positive tests post discharge with positive anal swabs. We report that $15.7 \%$ of patients $<50$ years old showed positive tests, while $2.4 \%$ of patients $>50$ years old showed positive tests from anal swabs. The delay between discharge and RT-PCR result date was negatively associated among positive cases of throat swabs, with an adjusted OR of 0.36 (95\% CI, 0.18-0.72). This finding implies that the risk of positive tests gradually vanishes over time.

Our study was limited by the lack of treatment information. Further and large-scale study on this phenomenon is warranted. Nevertheless, this study sheds lights on the viral dynamics of COVID-19.

Supplementary material. To view supplementary material for this article, please visit https://doi.org/10.1017/ice.2020.134 
Acknowledgments. We thank the Luohu Center for Disease Control (CDC), Futian CDC, Baoan CDC, Nanshan CDC, Longgang CDC, Yantian CDC, Longhua CDC, Guangming CDC, Pingshan CDC, Dapeng CDC, Shenzhen Samii Medical Center and Shenzhen Hezheng Hospital, all in Shenzhen, China, for offering 14-day isolation services for the discharged patients. We thank the Third People's Hospital of Shenzhen for the admission and treatment for the relapse patients.

Financial support. D.H. was supported by General Research Fund (grant no. 15205119) of Research Grants Council of Hong Kong and an Alibaba (China)-Hong Kong Polytechnic University Collaborative Research project. The funding agencies had no role in the design and conduct of the study; collection, management, analysis, and interpretation of the data; preparation, review, or approval of the manuscript; or decision to submit the manuscript for publication.

\section{References}

1. China National Health Commission. Diagnosis and treatment of the novel coronavirus (2019-nCoV) pneumonia in China [in Chinese]. China National Health Commission website. http://www.nhc.gov.cn/yzygj/s7653p/202002/ d4b895337e19445f8d728fcafle3e13a.shtml. Published 2020. Accessed April 16, 2020.

2. Lan L, Xu D, Ye G, et al. Positive RT-PCR test results in patients recovered from COVID-19. JAMA 2020. doi: 10.1001/jama.2020.2783.

3. Report of the WHO-China Joint Mission on Coronavirus Disease 2019 (COVID-19) from 16-24 February 2020. World Health Organization website. https://www.who.int/docs/default-source/coronaviruse/who-china-joint-missionon-covid-19-final-report.pdf. Published 2020. Accessed April 15, 2020.

4. Guan WJ, Ni ZY, Hu Y, et al. Clinical characteristics of coronavirus disease 2019 in China. N Engl J Med 2020. doi: 10.1056/NEJMoa2002032.

Conflicts of interest. D.H. was supported by an Alibaba (China)-Hong Kong Polytechnic University Collaborative Research project. All other authors declared no competing interests related to this article.

\title{
Pasteurized blood samples for transfusion compatibility testing during the coronavirus disease 2019 outbreak
}

\author{
Run Yao MS ${ }^{1}$, Yamei Shen BS ${ }^{1}$, Ying Tan MD, Pengcheng Zhou MD², Bijuan Li PhD¹, Xuegong Fan PhD²,3 and Ning Li PhD \\ ${ }^{1}$ Department of Blood Transfusion, Xiangya Hospital, Central South University, Changsha, 410007, Hunan, China, ${ }^{2}$ Department of Infectious Diseases, Xiangya \\ Hospital, Central South University, Changsha, China and ${ }^{3}$ Key Laboratory of Viral Hepatitis, Hunan Province, Changsha, China
}

To the Editor-In December 2019, a novel coronavirus pneumonia (COVID-19) was reported in Wuhan, China. As of April 2, 2020, 82,774 confirmed cases had been reported in China and 874,995 confirmed cases had been reported in other countries. No vaccine or antiviral therapeutics are yet available to prevent or treat COVID-19. ${ }^{1}$ Preventing infection is the current priority for disease control.

The SARS-CoV-2 virus is transmitted from person to person through droplets or direct contact. ${ }^{2}$ However, non-respiratory samples are also potential sources of COVID-19 infection. ${ }^{3}$ Virus-laden aerosols generated from blood-sample centrifugation pose risks for laboratory staff and broader nosocomial transmission. ${ }^{3,4}$ Traditional precautionary measures for infectious-sample processing include tertiary protection and operating in the biological safety cabinet. Preventive resources have been limited during this multiregional outbreak, posing huge risks to laboratory staff. Therefore, effective methods to ensure the safety of laboratory staff in low-resource settings are needed.

Pasteurization at $56^{\circ} \mathrm{C}$ for 30 minutes has been recommended to inactivate coronavirus, which might decrease the infectivity of samples and aerosols. To reduce infections and ensure safe and effective transfusion, we investigated the effects of pasteurization on transfusion compatibility testing.

Author for correspondence: Li Ning, E-mail: liningxy@csu.edu.cn

Cite this article: Yao R, et al. (2020). Pasteurized blood samples for transfusion compatibility testing during the coronavirus disease 2019 outbreak. Infection Control \& Hospital Epidemiology, 41: 1112-1114, https://doi.org/10.1017/ice.2020.138

\section{Methods}

Blood samples were collected from Xiangya Hospital, Central South University. Each sample was divided into 2 groups, an experimental group and a control group. Experimental samples were treated by pasteurization. The results of blood-group typing, irregular antibody screening, and cross-matching were compared between these 2 groups. Finally, samples of suspected SARS-CoV-2 were treated with pasteurization. Treated samples were used to test transfusion compatibility. Patients with suspected COVID-19 then received red blood cell (RBC) transfusion, and the effectiveness and safety of these transfusion were evaluated.

\section{Results}

The agglutination intensities of $\mathrm{A}, \mathrm{B}$ antigens and anti-A, anti-B antibodies of the samples in the 2 groups were $4+$. The forward and reverse types were consistent in the ABO blood group. In the $\mathrm{Rh}$ blood group, the agglutination intensity of $\mathrm{D}$ antigen was reduced from $4+$ to between $2+$ and $3+$ after heat treatment (Fig. 1). Regarding the effect of heat treatment on irregular antibody screening, our results showed that the response pattern of panel cells remained unchanged after heat treatment when the agglutination intensity was negative(-), uncertain( \pm ) or zero, and $1+, 2+$, or $3+$, respectively. However, the agglutination intensities of samples rating $4+$ were reduced to $3+$ after heat treatment (Fig. 2). Finally, no effect of heat treatment on the primary crossmatching was observed.

Our results indicated that heat treatment did not affect the results of transfusion compatibility testing. The RBC transfusion 